\title{
USAGE OF PROCESS MINING IN THE 'OFFER TO PRODUCTION PROCESS' OF A CONTRACT MANUFACTURER FOR CAST COMPONENTS
}

\author{
Jens Kiefer and Melina Precht \\ University of Applied Sciences Ulm \\ Prittwitzstraße 10, 89075 Ulm, Germany
}

\begin{abstract}
This paper presents potentials and current limits of the big data method process mining within the 'offer to production process' of a contract manufacturer for cast components. The considered process includes all essential activities from the offer inquiry to the production start of the ordered cast components. As a first step, the general goals and characteristics of the trendsetting method process mining are illustrated. After explaining the most important contents of the considered 'offer to production process', the usage of process mining within this topic field is demonstrated. Apart from methodical issues, information-technical and organizational aspects are discussed. In this context, one special focus is on the description of the required data preparation process, which forms one of the most significant fundamentals for an efficient and profitable execution of process mining. By means of a real existing practical example, the procedure, potentials and current limits of process mining using the software tools LANA and Celonis are critically evaluated. Finally, the paper ends with a conclusion and an outlook towards further research and development activities.
\end{abstract}

\section{KEYWORDS}

Process Mining, Data Management, Application

\section{PROCESS MINING}

According to van der Aalst (2016), process mining is an increasingly important and trendsetting method in the area of data science and big data approaches. In general, the goal of process mining is to gain information about real processes (e.g. production processes or administrative processes) based on real existing data stored in file- or database-based IT systems. Whereas classical data mining techniques are mostly data-centric, process mining is process-centric (Hand, 2001). The method process mining enables business processes to be reconstructed and analyzed based on 'digital traces' in IT systems. During process mining, specific data mining algorithms are applied to event log data in order to identify trends, patterns or details contained in event logs recorded by an information system (van der Aalst, 2011).

As illustrated in Figure 1, event logs can be used to conduct three types of process mining. The first type of process mining is discovery. The discovery technique aims to generate a process model based on log data without using any a-priori information. Currently, process discovery is the most popular process mining technique. The second type of process mining is conformance. The conformance technique compares an available process model with $\log$ data of the real process. As a result, the deviations between $\log$ data and process model are detected and visualized. Last and least, there is the enhancement technique. This approach enables the extension of an available process model by means of existing log data. Whereas the conformance technique measures the alignment between model and reality, this technique aims at extending or improving the a-priori model (e.g. addition of waiting times). 


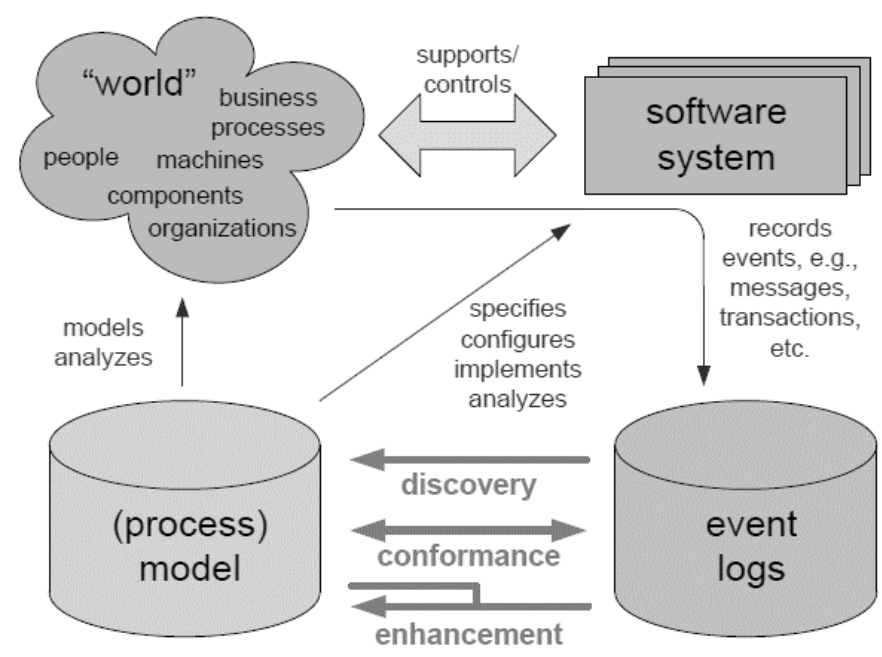

Figure 1. The three basic types of process mining: discovery, conformance and enhancement (van der Aalst, 2012)

According to van der Aalst (2016), process mining applications can be differentiated into three different ways: continuously, repeated and $a d-h o c$. The continuous application is appropriate for processes that shall be monitored on daily basis or in real time. Its implementation is quite expensive and only specific analyses can be executed because they must be implemented in the tool before. In contrast, the ad-hoc usage implies less effort during the implementation. This application is suitable for one-time analysis, which are never or seldom repeated. The ad-hoc usage is appropriate for unique optimizations and the detection of weaknesses in the process. The repeated application is located somewhere between the continuous and the ad-hoc solution. It is appropriate for analyzing processes e.g. on monthly basis or if the analysis only vary slightly.

This paper presents the usage of process mining focusing on process discovery and on ad-hoc analyses of the process data. Taking the real-existing application scenario 'offer to production process' of a contract manufacturer for cast components, potentials and current limits of process mining are portrayed and critically evaluated.

\section{APPLICATION SCENARIO AND CHALLENGES}

The process discussed in this contribution is located in the foundry sector. A contract manufacturer produces cast components - both ready-to-install parts and unfinished components - for various customers of different industries. Figure 2 illustrates the most important phases and their interrelations of the considered 'offer to production process'.

Before an offer inquiry of a customer is received, there is a series production of other products running. In parallel to this series production, the 'offer to production process' for new products is performed. This process starts with an incoming offer inquiry from a customer. After extensive planning steps such as the planning of the foundry process with the necessary production equipment as well as the corresponding logistics activities, an offer is presented to the customer. If the customer decides to accept this offer, the order allocation takes place. The incoming order represents the beginning of further activities: As a first step, the equipment for the casting process such as models or core boxes have to be planned in detail and manufactured. Afterwards, the production of the initial sample follows. If the customer accepts this sample, the Start of Production (SOP) enables the series production.

Due to recent developments the process of production planning is getting more and more complex. Customers tend to request ready-to-install components instead of unfinished parts. This development complicates the planning process significantly because there are only limited possibilities for further processing within the regarded company. The increasing customer expectations on the one hand and the rising number of requested products on the other hand lead to the necessity to gain more detailed knowledge about the actually lived processes as crucial basis to implement a KPI-driven continuous improvement process. 


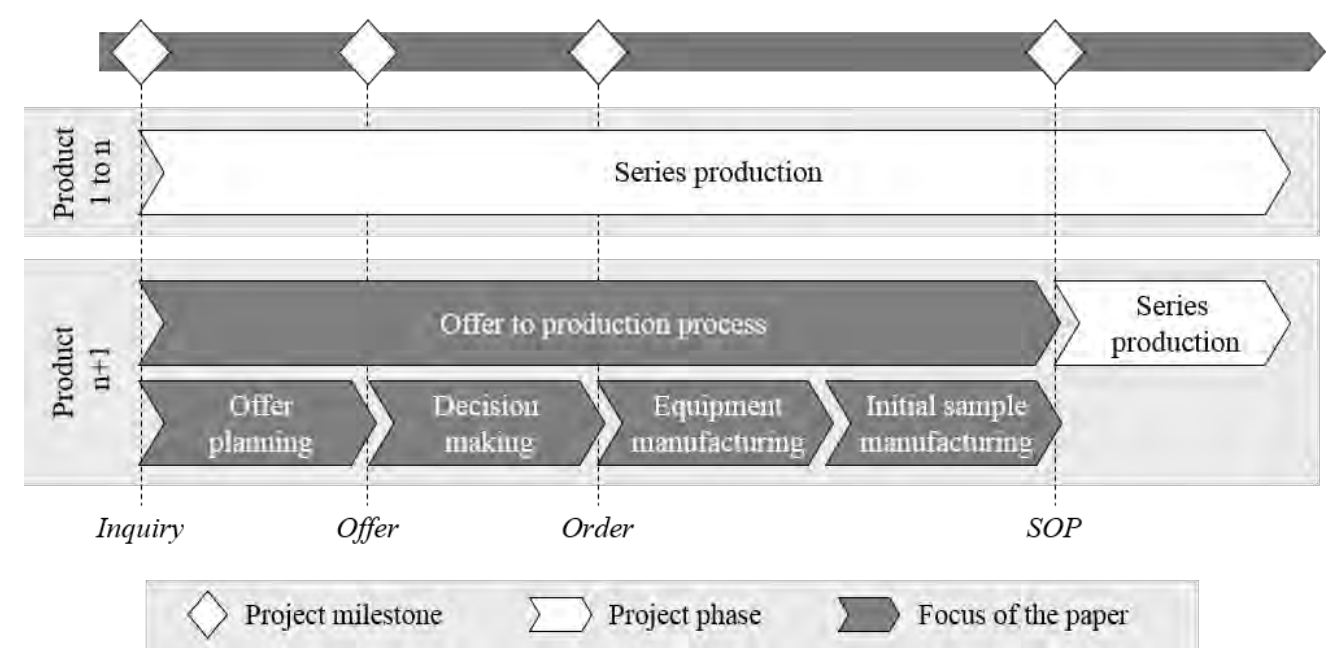

Figure 2. Simplified 'offer to production process' of a contract manufacturer

Within this application example, there are some specific challenges regarding the usage of process mining. At present, the most important challenges are:

- Process has a project character (e.g. different departments are involved in each offer planning process)

- Each subprocess (e.g. 'offer planning') contains several standard activities that are logically structured over a superior network (hereby, each standard activity can optionally be skipped)

- The 'offer planning' process consists of many activities that are conducted in parallel

- No concrete reference process due to high product and process variances

- Heterogeneous data sources (data are available in databases and file-based IT systems)

- Combination of automatically generated data and manual entered data

- About $30 \%$ of the available data sets are incomplete

- Process model consists of different process sequences (e.g. not all offers result in an order)

\section{METHODICAL IMPLEMENTATION}

There are several preparation steps necessary before an analysis with process mining is possible. The most important steps are:

1. Selecting the right data - deep know-how about the available data is necessary

2. Rating the individual data sources maturity level according to the Process Mining Manifesto (van der Aalst et al, 2012) - shows the suitability of each source for the implementation of process mining

3. Generating a data model of the considered objects (e.g. an inquiry or a requested component) including their relations, the individual identification and attributes - the current connections and connection possibilities between objects and different data sources become clear

4. Merging the heterogeneous data sources - to form a unified data set

The maturity level rating in the Process Mining Manifesto (van der Aalst et al, 2012) rates the maturity level of ' 5 ' as the best case and the maturity level of ' 1 ' as the worst case in the light of applying process mining. In general, a maturity level of ' 5 ' should be aimed for using process mining but the usage of data sources with a maturity level of ' 4 ' or ' 3 ' can mostly also be used.

In the considered 'offer to production process', there are six different data sources (e.g. a CSV file or the SAP SP-module), which have to be rated first according to the defined maturity levels. Table 1 illustrates the result of this rating process. This analysis shows that most of the data sources are generally suitable for the implementation of process mining. 
Table 1. Maturity levels of the used data sources

\begin{tabular}{|c|c|}
\hline Maturity level & Number of sources \\
\hline 5 & 0 \\
\hline 4 & 1 \\
\hline 3 & 3 \\
\hline 2 & 1 \\
\hline 1 & 1 \\
\hline
\end{tabular}

Due to the variety of the data sources that are used in the regarded process, the isolated rating of sources is not enough to specify the suitability for the process mining implementation. The combination of data sources must be considered additionally according to the specific process. In addition to that, the data quality must be rated in detail. Only if the data sources, the data relations and the data quality are known and good enough, so that a merging of the data sources is possible, an implementation of process mining is reasonable conceivable.

Figure 3 illustrates the developed data model regarding the merging process of the used heterogeneous data sources. When checking for connection possibilities, it is necessary that the individual object identifications can somehow be connected to the selected Case-ID. This connection can result from a third table, which owns both identifications (cp. Figure 3: 'Table'). A connection can also result from a data source that has one explicit identification and the identification of another data source as an extra attribute (cp. Figure 3: 'Data source 3'). If it is not possible to find a connection between all used data sources, the application of process mining is not possible for this case or another Case-ID must be considered.

In the considered 'offer to production process' a relation table is created as visualized in Figure 3 . With this table it is possible to assign the Case-ID to each data set by using the possessed identification as a connecting element. After the heterogeneous data forms a unit, the following implementation of process mining is possible.

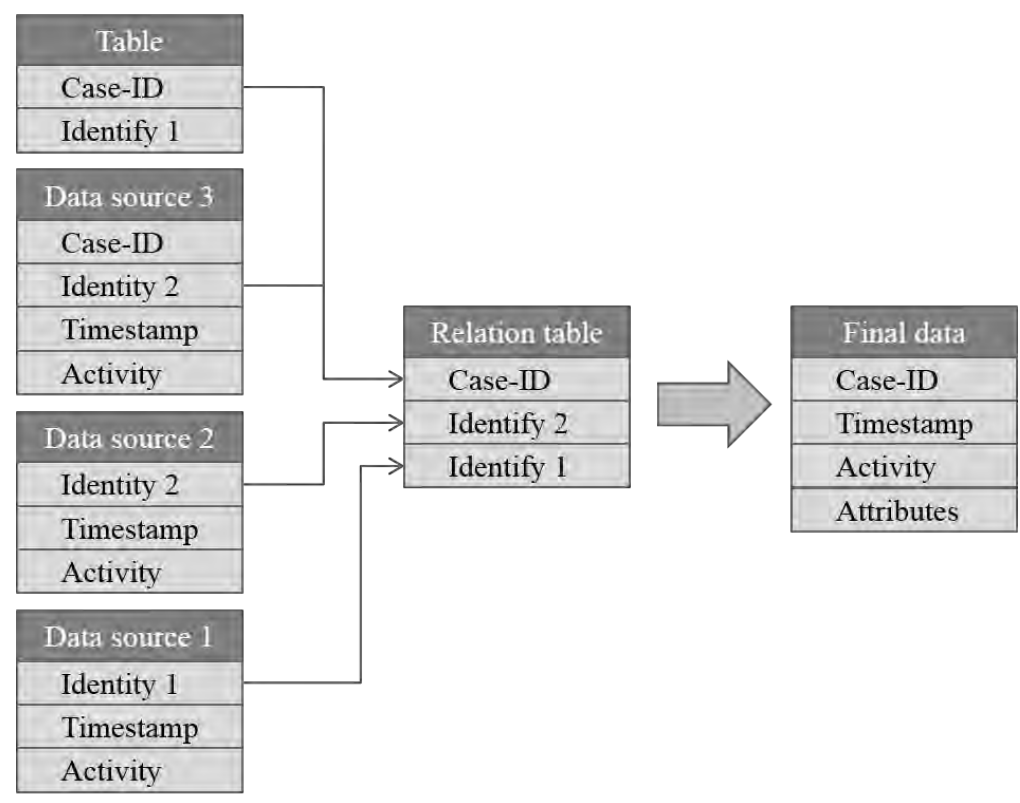

Figure 3. Data model of the merging process

The 'Final data' can now be played into the process mining tool. The tool arranges the activities according to the Case-ID and on basis of the timestamps. Thus, a process model is created, which displays how the single cases went through the process. First, the process model is chaotic and consists out of a wide range of activities (in this case up to 600 different activities are involved). If there are known filters to select the desired process, they are applied right away. Another possibility to gain model transparency is to reduce the visualized activities and paths between activities. However, this reduction would lead to information loss 
at the same time, because not all activities and paths that are executed are being displayed. To keep visualizing the complete process and still gaining clarity, several activities should be combined into one activity group. In order to carry this bundling process out as efficient as possible, this bundling should be based on logical aspects. Finally, the grouping of activities leads to a more transparent and clearer structured process model compared to the initial process model.

\section{INFORMATION-TECHNICAL IMPLEMENTATION}

\subsection{Used Process Mining Tools}

The information-technical implementation of the new developed process mining method was executed by using two different software tools: LANA and Celonis. LANA is a process mining tool from the Lana Labs $\mathrm{GmbH}$, which was developed in 2016 as spin-off from the Hasso-Platter-Institute. Celonis was formed in 2011 and is currently the global leader of process mining tools (Peters and Nauroth, 2019). Both software products were used to analyze the considered 'offer to production process'. In this context, the focus was set on the process discovery, the conformance technique and the execution of ad-hoc analyses. Even though the method of process mining is the same, there are some differences in the application of these two software tools.

The process model generated with LANA is clear in the visualization. One branch in the model displays an exclusive gateway. However, in the current software version it is not possible to visualize parallel activities. The activity sequence is based on the start timestamps. LANA calculates the waiting time between activities by considering the start of the predecessor and the start of the successor activity. The run time of an activity is calculated by forming the difference between end and start timestamp. In case of parallel processes, this calculation procedure leads to misleading results.

For processes that do not consist out of parallel activities, the visualization in LANA is easy to understand and specific. If there are parallel activities involved in the process, the process model must be analyzed with caution. In the considered process it is known that there are no waiting times between the single activities for production planning because they are controlled over a workflow. In this case, the discovered bottlenecks in the waiting times can be ignored. The displayed follow-connections can't always be taken as the reflection of reality.

In contrast to LANA, Celonis chose a process visualization that is not specific. A branch in the process model can be an exclusive gateway and a parallel gateway at the same time. That's the reason why the frequencies on the paths don't always match up with the frequencies of the predecessors and the successors. However, in the considered process, the ability of the used process mining tool to visualize and analyze parallel paths is very important. That's why the usage of Celonis for process discovery fits better for the considered 'offer to production process' than the LANA solution. In this context it can be noted, that there are many processes that consist of parallel activities. E.g. the product creation process includes a high number of parallel planning steps, which is rising due to recent developments to reduce the time to market. The inability of a process mining tool to discover and display parallel processes presents an essential difficulty in these cases.

Apart from the differences in the ability to visualize parallel processes, these two software tools have similar properties. LANA tends to offer less options, e.g. when trimming the process to a specific extract or in terms of possible KPI monitoring. However, the usage is very intuitive and especially for 'process mining beginners' LANA is easy to use. Celonis offers a broad range of additional analysis possibilities, which make it possible to create individual dashboards to monitor KPIs or other evaluations. LANA provides a set kind of evaluations, which satisfied most expectations in the regarding process. In general, it can be set out that LANA offers less freedom in adjusting the analysis technique than Celonis does. 


\subsection{Analysis of the Considered Process using Celonis}

In Figure 4, the discovered process model of the regarded process - with grouped activities - is visualized by using the process mining tool Celonis. The different shades in the background underline the former different data sets.
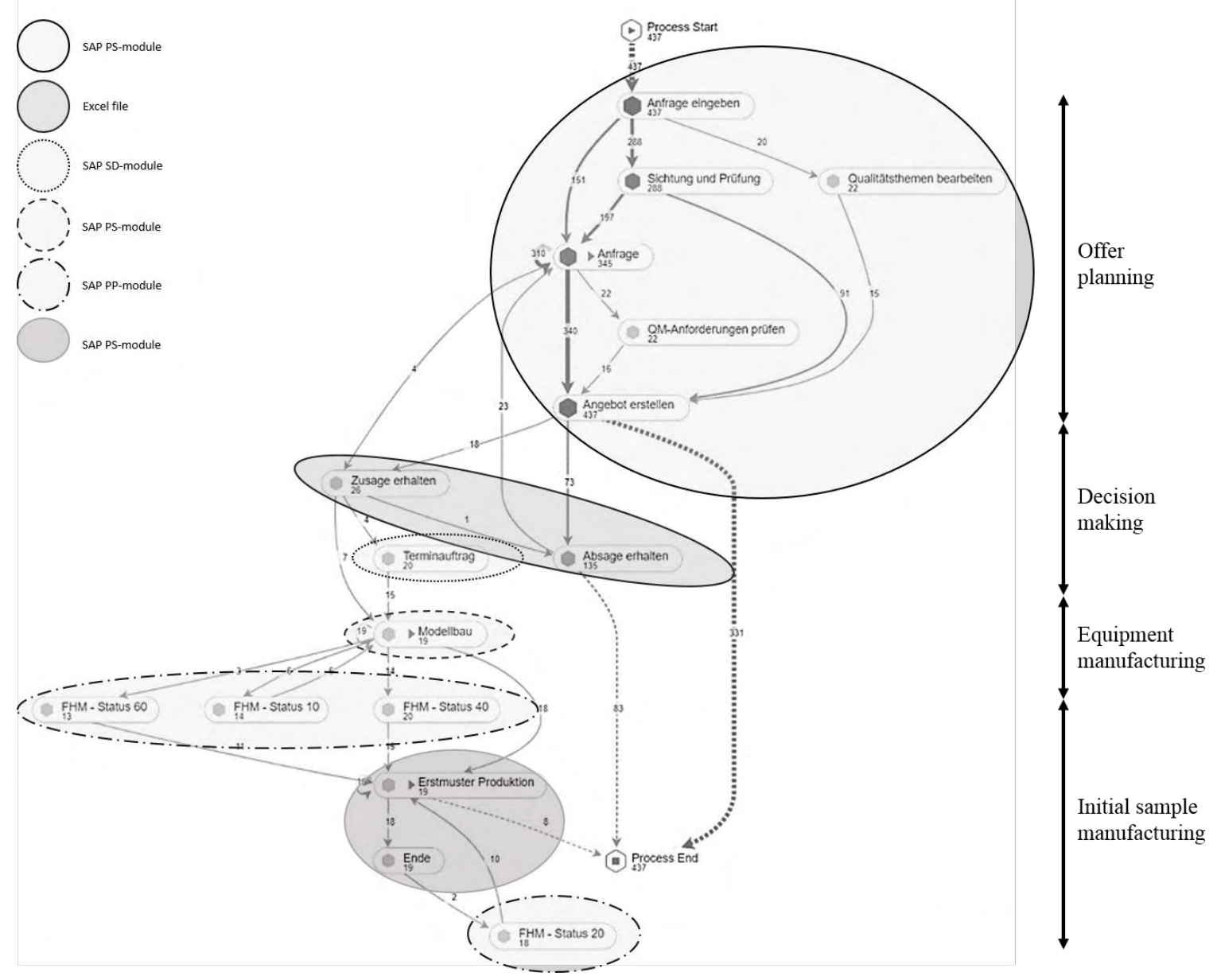

Figure 4. Process model (with logical groups) generated with Celonis

As shown in Figure 4, six data sources were used as basis for the analysis. The four subprocesses 'offer planning', 'decision making', 'equipment manufacturing' and 'initial sample manufacturing' (cp. Figure 2) are mapped in the used SAP PS-module with several activities. This module makes it possible to give a structure to a project by developing a project structure plan with several activities and to control the execution of these via a superior network. In addition to that, one data source origins from the SAP PP-module and another data source origins from the SAP SD-module. These data sources display the date of the query as well as some status changes of the casting equipment during the production of the initial sample. As part of an additional Excel file, the dates of the confirmation or rejection of the offer are documented, which were originally received via e-mail.

The process model in Figure 4 states a starting point for an analysis. The most common activities and paths are being displayed. As a next step, every process sequence can be analyzed in detail. Hereby, the focus is set on deviations from the expected behavior. For example, the rejection of an offer that is followed by further planning steps is an unwanted deviation from the process flow. Deep investigations about this sequence and the affected cases are necessary, which are supported by the process mining tool. In this context, diverse filter can be set to restrict the displayed model. Consequently, only the process cases are selected to form a model, that are relevant for the specific investigation. Furthermore, each particular case 
can be examined up to the single activities, their timestamps and attributes. This approach allows to find anomalies and to earn know-how about lead and waiting times of the process based on system data. This so called 'drill-down' is especially important because it starts by analyzing the big picture in which anomalies become clear that happen in a conspicuous frequency. As a next step, it is possible to look at explicit cases. In this context, it is possible to discuss the anomalies with the involved department, not only on KPIs or compromised data, but on real cases that are descriptive. This new possibility to state facts portrays the relevant characteristics of this new approach.

If there is a reference model that specifies the process sequence, this model can be imported into or created within the tool in form of a BPMN diagram. By using the conformance technique, the software tool compares the specified process model and the determined process model and visualizes the deviations between these two data models. However, this direct comparison between model and real data is only possible in parts of the regarded process, because many subprocesses have a project character and don't follow a certain target process.

\section{CONCLUSION}

This paper presents the usage of process mining within the 'offer to production process' of a contract manufacturer for cast components. By means of a real existing practical example, the application of this modern data mining technique - focusing on process discovery and on ad-hoc analyses - is illustrated. Apart from all the portrayed advantages and potentials of using process mining within this process, there are currently some limits and challenges, which have to be considered before introducing process mining into the operational practice.

The visualization of the process is only helpful, if it is possible to overlook the resulting process model. At first, this was not possible with up to 600 different activities displayed. The grouping of activities resolved this issue but also lead to an information loss because the connections between the grouped activities cannot be visualized and analyzed any more. If a process is supposed to be analyzed with process mining, it is recommended to choose either a shorter process or to reduce the activities' degree of detail.

The analysis of a process that has a project character and doesn't have to follow a certain target process is not ideal for the application of process mining. During the analysis some deviations can be worked out if the analyst has a deep process know-how or is even a process participant. Hence, it is certainly easier to be able to apply the conformance technique and visualize all deviations at once.

The variance in the process sequences is a further barrier. If the considered process shows a great variance in the length of the process sequence and amount of activities, a common analysis is difficult. Furthermore, the seldom occurrence of the desired path states a problem because process mining tends to hide seldom occurring paths and activities to keep the visualization simple and concisely. In this case, an alternative approach might be to separate the process sequences into two analyses. Hereby, the process sequences that stop after making an offer (no reply from the customer/ rejection of the offer) and the sequences that continue with further process steps (manufacturing of the casting equipment and initial samples) are displayed separately.

A further challenge refers to the presence of multiplex concurrent activities to be set out. Multiplex concurrencies occur especially in complex processes when several departments work on one product at the same time. According to Ly (2012), these concurrencies state a difficulty for any process discovery algorithms.

The listed challenges show what kind of process may have problems but at the same time gives an advice, which processes are ideal for being analyzed with process mining. Subsequently, general factors that have an impact on the application of process mining can be addressed. Hereby, the data analysis before the process mining implementation is essential. In this context, not only the data itself is relevant, but especially the analysis of the data quality.

In terms of data quality, it must be known if the data is complete, trustworthy and if the identification attributes have a reliable syntax. If the data that is going to be analyzed is incomplete, the evaluations are not reliable. If it is unknown that the data that is going to be analyzed is incomplete, conclusions might be drawn that are incorrect. Regardless of the type of analysis that is applied on data, the real population must be known so that the data input can be classified. The content of the data must also be investigated. If data is 
used, that is not trustful, it can't be expected to gain analysis results that are better than the input data. Since the method completely relies on the input data, the focus should be set on the data before doing an analysis. The goal of process mining to visualize the real process is only possible, if the data represents the reality. Therefore, the best data for process mining is produced by automated systems or machines instead of manual data input. The syntax of the data is another essential factor that must be considered. Especially if heterogeneous data sources are used for the analysis, the connecting elements for the allocation of the Case-ID must have an explicit syntax. If they don't, data loss is the consequence.

\section{OUTLOOK}

Dealing with data in general and the impact of process mining in particular largely depends on process and organizational aspects of a company. The economic impact of process mining rises and falls with the integration of process mining in a higher-level process management. Therefore, the aim of companies should be to integrate process mining as part of a seamless and consistent process management. This integration should be carried out in a holistic way taking into account the overall socio-technical system, which consists both of methodical, (information-)technical, organizational and human aspects (Netland et al, 2008). There are different approaches how to integrate process mining in an overall process management. One exemplary integration framework is described in Günther et al (2008). In addition to these technical integration challenges, the integration of process mining in the operational and organizational company and the associated organizational adaptations also play a significant role in the success of introducing process mining (Zhao and Zhao, 2014).

\section{REFERENCES}

Günther, C. et al, 2008. Using process mining to learn from process changes in evolutionary systems. In Int. J. Business Process Integration and Management, Vol. 3, No. 1.

Hand, D. et al, 2001. Principles of Data Mining. In MIT press, Cambridge, MA.

Ly, T. et al, 2012. Data Transformation and Semantic Log Purging for Process Mining. In Advanced Information Systems Engineering. CAiSE 2012. Lecture Notes in Computer Science, Vol. 7328, Springer, Berlin, Heidelberg.

Netland, T. et al, 2008. The new importance of socio-technical systems research on high-tech production systems. In Proceedings of the HOPS Conference 2008. Lausanne, Switzerland.

Peters, R. and Nauroth, M., 2019. Process-Mining Geschäftsprozesse: smart, schnell und einfach. Springer Gabler, Wiesbaden.

Van der Aalst, W., 2011. Process Mining - Discovery, Conformance and Enhancement of Business Processes. Springer-Verlag, Springer, Berlin, Heidelberg.

Van der Aalst, W., 2012. Process Mining: Overview and Opportunities. In ACM Transactions on Management Information Systems, Vol. 99, No. 99.

Van der Aalst, W. et al, 2012. Process Mining Manifesto. In Business Process Management Workshops. BPM 2011. Lecture Notes in Business Information Processing, Vol. 99, Springer Berlin, Heidelberg.

Van der Aalst, W., 2016. Process Mining - Data Science in Action. Springer-Verlag, Heidelberg.

Zhao, W. and Zhao, X., 2014. Process Mining from the Organizational Perspective. In Foundations of Intelligent Systems. Advances in Intelligent Systems and Computing, Vol. 277, Springer, Berlin, Heidelberg. 